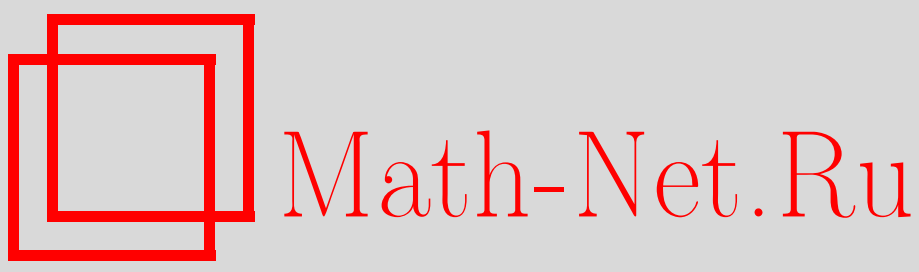

Е. Н. Сосов, О действиях с сохранением пучка прямых на пространстве Лобачевского групп вещественных чисел, Изв. вузов. Матем., 2019, номер 3, 32-37

DOI: https://doi.org/10.26907/0021-3446-2019-3-32-37

Использование Общероссийского математического портала Math-Net.Ru подразумевает, что вы прочитали и согласны с пользовательским соглашением

http://www . mathnet.ru/rus/agreement

Параметры загрузки:

IP: 18.234 .197 .8

26 апреля 2023 г., $17: 38: 09$

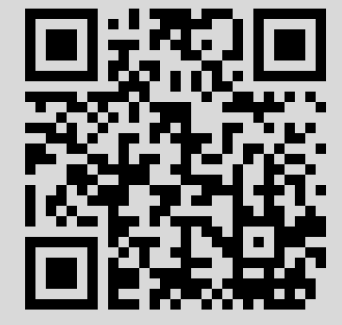


Известия вузов. Математика 2019, № 3, с. 32-37 https://kpfu.ru/science/nauchnye-izdaniya/ivrm/

e-mail: izvuz.matem@kpfu.ru

\title{
E.H. COCOB
}

\section{О ДЕЙСТВИЯХ С СОХРАНЕНИЕМ ПУЧКА ПРЯМЫХ НА ПРОСТРАНСТВЕ ЛОБАЧЕВСКОГО ГРУПП ВЕЩЕСТВЕННЫХ ЧИСЕЛ}

\begin{abstract}
Аннотация. Рассматривается модель Бельтрами-Клейна пространства Лобачевского $\Lambda$. Получены явные формулы для действий с сохранением гиперболического пучка прямых на пространстве $\Lambda$ мультипликативной группы ненулевых вещественных чисел и действий, сохраняющих параболический пучок прямых, аддитивной группы вещественных чисел.
\end{abstract}

Ключевые слова: пространство Лобачевского, модель Бельтрами-Клейна, пучок прямых.

УДК: 514.753

DOI: 10.26907/0021-3446-2019-3-32-37

\section{ВВЕДЕНИЕ}

Пусть $\mathbb{E}$ - полное, сепарабельное евклидово пространство над полем вещественных чисел $\mathbb{R}$ размерности $\operatorname{dim} \mathbb{E}>1$ и $B(O, 1) \subset \mathbb{E}$ - открытый шар с центром в фиксированной точке $O \in \mathbb{E}$ радиуса 1 . Точки пространства будем задавать их радиусами-векторами относительно точки $O$, которая при этом задается нулевым радиусом-вектором 0.

Рассмотрим модель Бельтрами-Клейна пространства Лобачевского [1]-[5]. В этой модели л-точка есть точка в $\Lambda=B(0,1)$, л-прямая есть хорда в $\Lambda$, а расстояние между л-точками $x, y \in \Lambda$ вычисляется по формуле [1]:

$$
\rho(x, y)=k \operatorname{Arch} \frac{1-(x, y)}{\sqrt{1-x^{2}} \sqrt{1-y^{2}}},
$$

где $k>0$ - константа, выбор которой равносилен выбору масштаба; $(x, y)$ - скалярное произведение радиусов-векторов точек $x, y \in \Lambda$ и $x^{2}$ - скалярный квадрат радиус-вектора точки $x$.

Пусть $\lambda \in \mathbb{R}, p, x \in \Lambda$. В [1] была определена точка $\lambda_{p}(x) \in \Lambda$ с помощью следующих трех условий:

1) $\rho\left(p, \lambda_{p}(x)\right)=|\lambda| \rho(p, x)$

2) если $x \neq p, \lambda>0$, то точки $x, \lambda_{p}(x)$ лежат на л-прямой $P(p, x)$, проходящей через точки $p, x$, по одну сторону от точки $p$.

$3)$ если $x \neq p, \lambda<0$, то точки $x, \lambda_{p}(x)$ лежат на л-прямой $P(p, x)$ по разные стороны от точки $p$.

Тогда отображение $x \mapsto \lambda_{p}(x)$, где $\lambda$ пробегает множество всех ненулевых вещественных чисел, определяет действие мультипликативной группы ненулевых вещественных чисел на пунктированном пространстве Лобачевского (точка $p \in \Lambda$ фиксирована), которое на каждой

Поступила в редакцию 11.01.2018, после доработки 07.03.2018. Принята к публикации 22.03.2018 
прямой эллиптического пучка является гомотетией и переводит в себя пучок концентричеких сфер с общим центром в точке $p$.

В той же работе (см. более подробно в [2]) было найдено следующее представление отображения $\lambda_{p}: \Lambda \rightarrow \Lambda$ :

$$
\lambda_{p}(x)=\frac{p \operatorname{ch} A \operatorname{sh}((1-\lambda) C)+x \operatorname{ch} B \operatorname{sh}(\lambda C)}{\operatorname{ch} A \operatorname{sh}((1-\lambda) C)+\operatorname{ch} B \operatorname{sh}(\lambda C)},
$$

где $A=\frac{\rho(0, p)}{k}, B=\frac{\rho(0, x)}{k}, C=\frac{\rho(p, x)}{k}$. В работе находятся аналогичные явные формулы для действий групп вещественных чисел на пространстве $\Lambda$, сохраняющих гиперболические и параболические пучки прямых. Это дает возможность получить различные расширения группы движений пространства Лобачевского, найти применения таких расширений в специальной теории относительности и фрактальной геометрии, получить специальные квазикомформные отображения пространства Лобачевского. В модели Пуанкаре в полупространстве евклидова пространства имеются просто устроенные параболический пучок прямых и пучок орисфер, поэтому с помощью изометрии моделей действие аддитивной группы вещественных чисел, сохраняющих эти пучки, переносится и в эту модель пространства Лобачевского.

\section{1. ОСНОВНЫЕ РЕЗУЛЬТАТЫ И ДОКАЗАТЕЛЬСТВА}

Пусть в пространстве $\Lambda$ заданы прямая $P$ и гиперплоскость П с уравнениями

$$
r=r_{0}+t a, \quad(n, x)=q,
$$

где $r_{0}$ - радиус-вектор фиксированной точки, $t-$ параметр, $a$ - направляющий вектор прямой $P, n$ - единичный вектор евклидовой нормали гиперплоскости $\Pi, 0 \leq q<1-$ константа. Сначала получим формулу для действия мультипликативной группы ненулевых вещественных чисел на пространстве $\Lambda$, сохраняющем гиперболический пучок прямых.

Теорема 1. Пусть в пространстве Лобачевского $\Lambda$ фиксированы прямая $P$, гиперплоскость П с уравнениями (3), а $x_{Q}$ - обозначение для ортогональной проекции по Лобачевскому произвольной точки $x \in \Lambda$ на $Q \in\{P, \Pi\}$. Тогда отображения

$$
\lambda_{Q}: \Lambda \rightarrow \Lambda, \quad \lambda_{Q}(x)=\frac{x_{Q} \operatorname{ch} A_{Q} \operatorname{sh}\left((1-\lambda) C_{Q}\right)+x \operatorname{ch} B \operatorname{sh}\left(\lambda C_{Q}\right)}{\operatorname{ch} A_{Q} \operatorname{sh}\left((1-\lambda) C_{Q}\right)+\operatorname{ch} B \operatorname{sh}\left(\lambda C_{Q}\right)},
$$

где $A_{Q}=\frac{\rho\left(0, x_{Q}\right)}{k}, B=\frac{\rho(0, x)}{k}, C_{Q}=\frac{\rho\left(x_{Q}, x\right)}{k}$, при $\lambda \in \mathbb{R} \backslash\{0\}$ определяют действие мультипликативной группы ненулевых вещественных чисел на $\Lambda$. Кроме того, $\lambda_{P}$ при каждом $\lambda \in \mathbb{R} \backslash\{0\}$ является гомотетией на каждой прямой, ортогональной прямой $P$, и переводит в себя пучок эквидистантных гиперповерхностей для прямой $P$ (так называемых гиперповерхностей Клифборда), а $\lambda_{\Pi}$ является гомотетией на каждой прямой гиперболического пучка прямых, ортогональной гиперплоскости П, и переводит в себя пучок эквидистантных гиперповерхностей с базой П.

Доказательство. Пусть $x \in \Lambda$. Тогда векторнопараметрическое уравнение прямой в пространстве $\mathbb{E}$, проходящей через точку $x$ и полюс гиперплоскости П, можно задать в форме

$$
r=x+t(q x-n),
$$

где $t$ - параметр. Пересечение этой прямой с гиперплоскостью П произойдет при значении параметра

$$
t_{*}=\frac{(n, x)-q}{1-q(n, x)} .
$$


Тогда ортогональная проекция $x_{\Pi}$ по Лобачевскому точки $x \in \Lambda$ на гиперплоскость П примет вид

$$
x_{\Pi}=\frac{\left(1-q^{2}\right) x+(q-(n, x)) n}{1-q(n, x)} .
$$

Рассмотрим теперь гиперплоскость с уравнением

$$
\left(R,(a, x) r_{0}+\left(1-\left(r_{0}, x\right)\right) a\right)=(a, x) .
$$

Очевидно, что она проходит через точку $x$ и ее полюс лежит на прямой $P$. Теперь нетрудно найти параметр $t_{1}$, который соответствует точке пересечения прямой $P$ с этой гиперплоскостью:

$$
t_{1}=\frac{\left(a,\left(1-r_{0}^{2}\right) x-\left(1-\left(x, r_{0}\right)\right) r_{0}\right)}{\left(a,(a, x) r_{0}+\left(1-\left(x, r_{0}\right)\right) a\right)} .
$$

Тогда ортогональная проекция $x_{P}$ по Лобачевскому точки $x \in \Lambda$ на прямую $P$ примет вид

$$
x_{P}=r_{0}+\frac{\left(a,\left(1-r_{0}^{2}\right) x-\left(1-\left(x, r_{0}\right)\right) r_{0}\right)}{\left(a,(a, x) r_{0}+\left(1-\left(x, r_{0}\right)\right) a\right)} a .
$$

Используя формулы (5), (6), представление (2) отображения $\lambda_{p}$ и его определение, получим представление (4). Оставшиеся свойства теперь очевидны.

Следствие. Пусть в пространстве Лобачевского $\Lambda$ фиксированы прямая $P$ и гиперплоскость П с уравнениями (3), где $r_{0}=0, q=0,|a|=1$. Тогда

$$
x_{P}=(a, x) a, \quad x_{\Pi}=x-(n, x) n
$$

и при $Q \in\{P, \Pi\}, x \in \Lambda$ имеем

$$
\lambda_{Q}(x)=x_{Q}+\left(x-x_{Q}\right) \operatorname{th}\left(\lambda C_{Q}\right) \operatorname{cth} C_{Q},
$$

где $C_{Q}=\frac{\rho\left(x_{Q}, x\right)}{k}$.

Теперь получим формулу для действия аддитивной группы вещественных чисел на пространстве $\Lambda$, сохраняющем параболический пучок прямых.

Теорема 2. Пусть единичный вектор $\tau$ евклидова пространства является радиус-вектором чентра параболического пучка в пространстве Лобачевского $\Lambda, h \in \mathbb{R}$. Тогда отображение $h_{\tau}: \Lambda \rightarrow \Lambda$,

$$
h_{\tau}(x)=\tau+\frac{2(\tau, \tau-x)}{(\tau-x)^{2}+\left(1-x^{2}\right) e^{\frac{-2 h}{k}}}(x-\tau)
$$

определяет действие аддитивной группь вещественных чисел на $\Lambda$ такое, что $\rho\left(x, h_{\tau}(x)\right)=|h|$ для каждого $x \in \Lambda$. Кроме того, $h_{\tau}$ на каждой прямой параболического пучка прямых с иентром в $\tau$ является параллельным переносом и переводит в себя пучок эквидистантных гиперповерхностей для такой прямой, а также пучок орисфер с центром в $\tau$.

Доказательство. Представим

$$
h_{\tau}(x)=\tau+t(x-\tau),
$$

где $t$ будем искать из условия $\rho\left(h_{\tau}(x), x\right)=|h|$. Тогда, используя $(1)$, получим уравнение

$$
\operatorname{ch} \frac{h}{k}=\frac{1-(x, \tau+t(x-\tau))}{\sqrt{1-x^{2}} \sqrt{1-(\tau+t(x-\tau))^{2}}}
$$


эквивалентная форма которого имеет вид

$t^{2}\left((x-\tau, x)^{2}+\operatorname{ch}^{2} \frac{h}{k}\left(1-x^{2}\right)(x-\tau)^{2}\right)-2 t(1-(\tau, x))\left(\operatorname{ch}^{2} \frac{h}{k}\left(1-x^{2}\right)+(x, x-\tau)\right)+(1-(\tau, x))^{2}=0$.

Найдем корни этого уравнения:

$$
\begin{aligned}
t_{1,2}=\frac{(1-(\tau, x))\left(\operatorname{ch}^{2} \frac{h}{k}\left(1-x^{2}\right)+(x, x-\tau)\right) \pm(1-(\tau, x))\left(1-x^{2}\right) \operatorname{ch} \frac{h}{k} \operatorname{sh} \frac{|h|}{k}}{\operatorname{ch}^{2} \frac{h}{k}\left(1-x^{2}\right)(x-\tau)^{2}+(x, x-\tau)^{2}}= \\
=\frac{(1-(\tau, x))\left(\left(1-x^{2}\right) e^{\frac{h}{k}} \operatorname{ch} \frac{h}{k}+(x, x-\tau)\right)}{\operatorname{ch}^{2} \frac{h}{k}\left(1-x^{2}\right)(x-\tau)^{2}+(x, x-\tau)^{2}}=\frac{2(\tau, \tau-x)}{(\tau-x)^{2}+\left(1-x^{2}\right) e^{\frac{-2 h}{k}} .}
\end{aligned}
$$

Таким образом, представление (7) верно. Представим радиус-вектор произвольной точки $x$ в виде $x=x_{1} \tau+y$, где вектор $y$ ортогонален $\tau$, и рассмотрим в $\Lambda$ прямую с уравнением $r=t \tau$. Эквидистантная гиперповерхность такой прямой имеет уравнение

$$
x_{1}^{2}+\frac{y^{2}}{a^{2}}=1,
$$

где $0<a<1$ - вещественная константа. Найдем уравнение прообраза этой гиперповерхности при отображении $h_{\tau}$

$$
\left(1+\frac{2\left(1-x_{1}\right)}{\left(1-x_{1}\right)^{2}+y^{2}+\left(1-x_{1}^{2}-y^{2}\right) e^{\frac{-2 h}{k}}}\left(x_{1}-1\right)\right)^{2}+\frac{4 y^{2}\left(1-x_{1}\right)^{2}}{a^{2}\left(\left(1-x_{1}\right)^{2}+y^{2}+\left(1-x_{1}^{2}-y^{2}\right) e^{\frac{-2 h}{k}}\right)^{2}}=1,
$$

его эквивалентная форма имеет вид

$$
x_{1}^{2}+\frac{\left.y^{2}\left(\left(1-a^{2}\right) e^{\frac{2 h}{k}}+a^{2}\right)\right)}{a^{2}}=1 .
$$

Таким образом, $h_{\tau}$ переводит в себя пучок эквидистантных гиперповерхностей для данной прямой. Любой другой пучок эквидистантных гиперповерхностей для прямой из параболического пучка с центром в $\tau$ можно получить из рассмотренного движением. Остальные утверждения теоремы теперь очевидны.

Пусть $n$ - единичный вектор евклидова пространства $\mathbb{E}$. Представим радиус-вектор произвольной точки $x$ этого пространства в виде $x=x_{1} n+x_{2}$, где вектор $x_{2}$ ортогонален $n$, и рассмотрим в полупространстве $\Pi_{+}=\left\{\left(x_{1} ; x_{2}\right) \in \mathbb{E}: x_{1}>0\right\}$ модель Пуанкаре с метрикой [2]:

$$
\rho_{\Pi_{+}}\left(\left(x_{1} ; x_{2}\right),\left(y_{1} ; y_{2}\right)\right)=k \operatorname{Arch} \frac{x_{1}^{2}+y_{1}^{2}+\left(x_{2}-y_{2}\right)^{2}}{2 x_{1} y_{1}}=2 k \operatorname{Arsh} \frac{|x-y|}{2 \sqrt{x_{1} y_{1}}} .
$$

Найдем явный вид действия аддитивной группы вещественных чисел в модели Пуанкаре $\left(\Pi_{+}, \rho_{\Pi_{+}}\right)$, сохраняющего параболический пучок прямых.

Теорема 3. Имеют место следующие утверждения.

а) Отображение

$$
\Phi:(\Lambda, \rho) \rightarrow\left(\Pi_{+}, \rho_{\Pi_{+}}\right), \quad \Phi\left(\left(x_{1} ; x_{2}\right)\right)=\frac{\left(\sqrt{1-x_{1}^{2}-x_{2}^{2}} ; x_{2}\right)}{1+x_{1}}
$$

является изометрией, обратная изометрия которого имеет вид

$$
\Phi^{-1}\left(\left(x_{1} ; x_{2}\right)\right)=\frac{\left(1-x_{1}^{2}-x_{2}^{2} ; 2 x_{2}\right)}{1+x_{1}^{2}+x_{2}^{2}} .
$$


b) Пусть

$$
g_{a}:(\Lambda, \rho) \rightarrow(\Lambda, \rho), \quad g_{a}\left(\left(x_{1} ; x_{2}\right)\right)=\frac{\left(x_{1}+p ; x_{2} \sqrt{1-p^{2}}\right)}{1+p x_{1}}
$$

- параллельный перенос на вектор $a=p n$, где $p=|a|<1$, в модели Бельтрами-Клейна [1]. Тогда в модели Пуанкаре при изометрии $\Phi$ ему соответствует преобразование

$$
G_{a}=\Phi \circ g_{a} \circ \Phi^{-1}:\left(\Pi_{+}, \rho_{\Pi_{+}}\right) \rightarrow\left(\Pi_{+}, \rho_{\Pi_{+}}\right), \quad G_{a}\left(\left(x_{1} ; x_{2}\right)\right)=\frac{\sqrt{1-p}}{\sqrt{1+p}}\left(\left(x_{1} ; x_{2}\right)\right) .
$$

с) Пусть $\tau=-n$. Отображсению $h_{\tau}: \Lambda \rightarrow \Lambda$, заданному формулой (7), в модели Пуанкаре при изометрии $\Phi$ соответствует преобразование

$$
H_{\tau}=\Phi \circ h_{\tau} \circ \Phi^{-1}:\left(\Pi_{+}, \rho_{\Pi_{+}}\right) \rightarrow\left(\Pi_{+}, \rho_{\Pi_{+}}\right), \quad H_{\tau}\left(\left(x_{1} ; x_{2}\right)\right)=\left(\left(e^{\frac{-h}{k}} x_{1} ; x_{2}\right)\right),
$$

которое определяет действие аддитивной группы вещественных чисел на $\Pi_{+}$такое, что $\rho_{\Pi_{+}}\left(x, H_{\tau}(x)\right)=|h|$ для каждого $x \in \Pi_{+}$. Кроме того, $H_{\tau}$ на каждой прямой параболического пучка прямых с направляющим вектором п является параллельным переносом и переводит в себя пучок эквидистантных гиперповерхностей для такой прямой (изображсаемых в модели Пуанкаре полугиперконусами вращения вокруг этой прямой с общей вершиной на абсолюте), а также пучок орисфер с уравнениями $x_{1}=$ const.

Доказательство. а) Покажем, что отображение $\Phi$ сохраняет расстояния между соответствующими парами точек

$$
\begin{aligned}
\operatorname{ch} \frac{\rho_{\Pi_{+}}\left(\Phi\left(\left(x_{1} ; x_{2}\right)\right), \Phi\left(\left(y_{1} ; y_{2}\right)\right)\right)}{k}= & \frac{\frac{1-x_{1}^{2}-x_{2}^{2}}{\left(1+x_{1}\right)^{2}}+\frac{1-y_{1}^{2}-y_{2}^{2}}{\left(1+y_{1}\right)^{2}}+\left(\frac{x_{2}}{1+x_{1}}-\frac{y_{2}}{1+y_{1}}\right)^{2}}{\frac{2 \sqrt{1-x_{1}^{2}-x_{2}^{2}} \sqrt{1-y_{1}^{2}-y_{2}^{2}}}{\left(1+x_{1}\right)\left(1+y_{1}\right)}}= \\
& =\frac{1-x_{1} y_{1}-\left(x_{2}, y_{2}\right)}{\sqrt{1-x_{1}^{2}-x_{2}^{2}} \sqrt{1-y_{1}^{2}-y_{2}^{2}}}=\operatorname{ch} \frac{\rho\left(\left(x_{1} ; x_{2}\right),\left(y_{1} ; y_{2}\right)\right)}{k} .
\end{aligned}
$$

Осталась нетрудная проверка того, что приведенная формула для $\Phi^{-1}$ действительно определяет обратное отображение.

b) Учитывая полученные формулы в п.а), с помощью непосредственного вычисления докажем

$$
\begin{gathered}
\Phi \circ g_{a} \circ \Phi^{-1}\left(\left(x_{1} ; x_{2}\right)\right)=\Phi \circ g_{a}\left(\frac{\left(1-x_{1}^{2}-x_{2}^{2} ; 2 x_{2}\right)}{1+x_{1}^{2}+x_{2}^{2}}\right)= \\
=\Phi\left(\frac{\left(\frac{1-x_{1}^{2}-x_{2}^{2}}{1+x_{1}^{2}+x_{2}^{2}}+p ; \frac{2 x_{2} \sqrt{1-p^{2}}}{1+x_{1}^{2}+x_{2}^{2}}\right)}{1+\frac{p\left(1-x_{1}^{2}-x_{2}^{2}\right)}{1+x_{1}^{2}+x_{2}^{2}}}\right)=\Phi\left(\frac{\left(1+p+(p-1)\left(x_{1}^{2}+x_{2}^{2}\right) ; 2 x_{2} \sqrt{1-p^{2}}\right)}{1+p+(1-p)\left(x_{1}^{2}+x_{2}^{2}\right)}\right)= \\
=\frac{\left(\sqrt{\left(1+p+(1-p)\left(x_{1}^{2}+x_{2}^{2}\right)\right)^{2}-\left(1+p+(p-1)\left(x_{1}^{2}+x_{2}^{2}\right)\right)^{2}-4 x_{2}^{2}\left(1-p^{2}\right)} ; 2 x_{2} \sqrt{1-p^{2}}\right)}{2(1+p)}= \\
=\frac{\sqrt{1-p}}{\sqrt{1+p}}\left(\left(x_{1} ; x_{2}\right)\right) .
\end{gathered}
$$

c) Сначала прямым вычислением найдем искомое преобразование

$$
\Phi \circ h_{\tau} \circ \Phi^{-1}\left(\left(x_{1} ; x_{2}\right)\right)=\Phi \circ h_{\tau}\left(\frac{\left(1-x_{1}^{2}-x_{2}^{2} ; 2 x_{2}\right)}{1+x_{1}^{2}+x_{2}^{2}}\right)=
$$




$$
\begin{aligned}
& =\Phi\left((-1 ; 0)+\frac{2\left(1+\frac{1-x_{1}^{2}-x_{2}^{2}}{1+x_{1}^{2}+x_{2}^{2}}\right)\left(1+\frac{1-x_{1}^{2}-x_{2}^{2}}{1+x_{1}^{2}+x_{2}^{2}} ; \frac{2 x_{2}}{1+x_{1}^{2}+x_{2}^{2}}\right)}{\left(1+\frac{1-x_{1}^{2}-x_{2}^{2}}{1+x_{1}^{2}+x_{2}^{2}}\right)^{2}+\left(\frac{2 x_{2}}{1+x_{1}^{2}+x_{2}^{2}}\right)^{2}+\left(1-\left(\frac{1-x_{1}^{2}-x_{2}^{2}}{1+x_{1}^{2}+x_{2}^{2}}\right)^{2}-\left(\frac{2 x_{2}}{1+x_{1}^{2}+x_{2}^{2}}\right)^{2}\right) e^{\frac{-2 h}{k}}}\right)= \\
& =\Phi\left(\frac{\left(1-x_{2}^{2}-x_{1}^{2} e^{\frac{-2 h}{k}} ; 2 x_{2}\right)}{1+x_{2}^{2}+x_{1}^{2} e^{\frac{-2 h}{k}}}\right)=\frac{\left(\sqrt{\left(1+x_{2}^{2}+x_{1}^{2} e^{\frac{-2 h}{k}}\right)^{2}-\left(1-x_{2}^{2}-x_{1}^{2} e^{\frac{-2 h}{k}}\right)^{2}-4 x_{2}^{2}} ; 2 x_{2}\right)}{2}= \\
& =\left(e^{\frac{-h}{k}} x_{1} ; x_{2}\right) .
\end{aligned}
$$

Теперь оставшиеся утверждения являются следствиями п. а), полученной формулы и теоремы 2 .

\section{ЛитеРАТУРА}

[1] Сосов Е.Н. О действии мультипликативной группь ненулевых вещественнъх чисел на пунктированном пространстве Лобачевского, Учен. зап. Казанск. ун-та. Сер. Физ.матем. науки 154 (4), 156-160 (2012).

[2] Сосов Е.Н. Геометрия Лобачевского и ее применение в специалъной теории относительности. Учебнометодическое пособие (Казанск. ун-т, Казань, 2016).

[3] Нут Ю.Ю. Геометрия Лобачевского в аналитическом изложении (Изд-во АН СССР, М., 1961).

[4] Трайнин Я.Л. Аналитическая геометрия в пространстве Лобаческого (Новосибирск. гос. педагогический ин-т, Новосибирск, 1974).

[5] Сабинин Л.В. Одули как новый подход к геометрии со связностью, ДАН СССР 233 (5), $800-803$ (1977).

Евгений Николаевич Сосов

Казанский федеральный университет,

ул. Кремлевская, д. 18, г. Казань, 420008, Россия,

e-mail: Evgenii.Sosov@kpfu.ru

\section{E.N.Sosov}

\section{On the real group actions preserving bundle of straight lines on the Lobachevskii space}

Abstract. We consider the Lobachevskii space. In terms of the Beltrami-Klein model we obtain explicit expressions for the real group actions preserving hyperbolic or parabolic bundle of straight lines on the Lobachevskii space.

Keywords: Lobachevskii space, Beltrami-Klein model, bundle of straight lines.

Evgenii Nikolaevich Sosov

Kazan Federal University,

18 Kremlyovskaya str., Kazan, 420008 Russia,

e-mail: Evgenii.Sosov@kpfu.ru 